\title{
Galsulfase (Naglazyme®) therapy in infants with mucopolysaccharidosis VI
}

\author{
Paul R. Harmatz • Paula Garcia • Nathalie Guffon • Linda M. Randolph • \\ Renée Shediac • Elizabeth Braunlin • Ralph S. Lachman • Celeste Decker
}

Received: 31 May 2013 /Revised: 28 August 2013 / Accepted: 11 September 2013 / Published online: 10 October 2013

(C) The Author(s) 2013. This article is published with open access at Springerlink.com

\begin{abstract}
Objective To evaluate the efficacy and safety of two dose levels of galsulfase (Naglazyme ${ }^{\circledR}$ ) in infants with MPS VI.

Study design This was a phase 4, multicenter, multinational, open-label, two-dose level study. Subjects were randomized $1: 1$ to receive weekly infusions of 1.0 or $2.0 \mathrm{mg} / \mathrm{kg}$ of galsulfase for a minimum of 52 weeks. Progression of skeletal dysplasia was determined by monitoring physical appearance, radiographic changes, and growth. Urinary glycosaminoglycan (GAG) levels, gross and fine motor function, cardiac function, vision, hearing, and health resource utilization were evaluated. Safety assessments were performed.
\end{abstract}

Communicated by: Gregory M. Pastores

Electronic supplementary material The online version of this article (doi:10.1007/s10545-013-9654-7) contains supplementary material, which is available to authorized users.

P. R. Harmatz $(\square)$

Children's Hospital \& Research Center Oakland, 747 52nd Street,

Oakland, CA, USA

e-mail:pharmatz@mail.cho.org

P. Garcia

Hospital Pediátrico de Coimbra, Coimbra, Portugal

N. Guffon

Hôpital Femme Mère Enfant, Lyon, France

L. M. Randolph

Children's Hospital Los Angeles, Los Angeles, CA, USA

R. Shediac $\cdot$ C. Decker

BioMarin Pharmaceutical Inc., Novato, CA, USA

E. Braunlin

University of Minnesota, Minneapolis, MN, USA

R. S. Lachman

International Skeletal Dysplasia Registry, Cedars-Sinai Medical

Center, Los Angeles, CA, USA
Results Four infants (aged 3.3-12.7 months) participated in the study. Galsulfase was well tolerated at 1.0 and $2.0 \mathrm{mg} / \mathrm{kg} / \mathrm{week}$ dose levels with no drug-related serious adverse events. Two subjects experienced a total of four possible treatment-related adverse events which were all considered mild. Length and weight remained within age-expected norms. Skeletal abnormalities continued to progress in all subjects. High baseline urinary GAG levels (mean: $870 \mu \mathrm{g} / \mathrm{mg}$ creatinine) decreased by approximately $70 \%$; these reduced levels were maintained (mean: $220 \mu \mathrm{g} / \mathrm{mg}$ creatinine at week 52) despite the development of anti-galsulfase antibodies. Hearing, cardiac function, hepatosplenomegaly, and facial dysmorphism stabilized or improved, but corneal clouding progressed. There was no clear difference in safety or efficacy between the two doses.

Conclusions Galsulfase at two dose levels was safe and well tolerated in infants. Normal growth was maintained but skeletal abnormalities continued to progress. Urinary GAG levels decreased with treatment. Early initiation of galsulfase may prevent or slow progression of some disease manifestations.

\section{Introduction}

Mucopolysaccharidosis VI (MPS VI, or Maroteaux-Lamy syndrome; OMIM 253200) is a lysosomal storage disorder caused by deficient activity of $N$-acetylgalactosamine 4sulfatase (arylsulfatase B or ASB; EC 3.1.6.12), the enzyme that catabolizes the glycosaminoglycan (GAG) dermatan sulfate. Affected individuals usually appear normal at birth, but intracellular GAG accumulation leads to progressive development of multisystemic clinical manifestations, including short stature, skeletal abnormalities, respiratory complications, cardiac disease, corneal clouding, hearing loss, spinal cord compression and reduced physical endurance (Giugliani et al 2007; Valayannopoulos et al 2010). Patients typically present with the radiographic changes of dysostosis multiplex, which comprise malformations of the skull, thorax, spine, pelvis, long 
bones and hands (Lachman et al 2010). There is wide variability in phenotypic presentation. Rapidly progressing patients usually begin to show symptoms shortly after birth and are typically diagnosed between 2 and 4 years of age (unless they have a family history). Most do not live to adulthood. Individuals with a slower disease course typically develop similar symptoms later in life (Thumler et al 2012). Urinary GAG levels $>200 \mu \mathrm{g} / \mathrm{mg}$ creatinine are generally associated with rapidly progressive disease while urinary GAG levels $<100 \mu \mathrm{g} / \mathrm{mg}$ creatinine are associated with a slowly progressing clinical course and longer survival (Swiedler et al 2005).

Galsulfase (Naglazyme ${ }^{\circledR}$ ), a recombinant human $N-$ acetylgalactosamine 4-sulfatase (rh-arylsulfatase B; rhASB), is currently approved by the United States Food and Drug Administration (US FDA), the European Medicines Agency (EMA) and regulatory agencies in several other countries as first-line therapy for MPS VI at an intravenous (IV) infusion dose of $1.0 \mathrm{mg} / \mathrm{kg} / \mathrm{week}$. Treatment with this dose of galsulfase has been shown to improve walking and stair-climbing capacity (Harmatz et al 2005). Enzyme replacement therapy (ERT) with galsulfase has also resulted in improvements in pulmonary function (Harmatz et al 2005, 2008), growth rate (Decker et al 2010), and pubertal development (Decker et al 2010). Approval of galsulfase as treatment for MPS VI was based on improvement in endurance in patients older than 5 years of age. Studies of two MPS VI sibling pairs, one from Australia (McGill et al 2010) and one from Japan (Furujo et al 2011), as well as a recent report from Brazil of 34 MPS VI children younger than 5 years (Horovitz et al 2013), suggest that initiation of ERT at an earlier age facilitates better outcomes, underscoring the need to evaluate the use of galsulfase in infants and very young children in controlled trials. Moreover, early intervention may have the potential to normalize bone growth and reduce the significant morbidity caused by skeletal involvement. Although results from both sibling studies suggest that early ERT at the $1 \mathrm{mg} / \mathrm{kg} /$ week dose does not prevent the progression of skeletal pathology in MPS VI infants and children under 5 years of age (Furujo et al 2011; McGill et al 2010), the effect of a higher dose of galsulfase has not been investigated. In MPS VIaffected cats, improvements in bone development as well as other MPS VI disease symptoms were associated with higher enzyme doses when ERT was initiated from birth (Byers et al 1997; Crawley et al 1997). Administration of a weekly $2 \mathrm{mg} / \mathrm{kg}$ dose would represent a significant increase in drug exposure as well as enable evaluation of safety of a higher dose.

The primary objective of this study was to evaluate the efficacy of two dose levels (1.0 and $2.0 \mathrm{mg} / \mathrm{kg} /$ week) of galsulfase on the progression of skeletal dysplasia (as reflected by changes in radiographs, physical appearance, and growth) in infants with MPS VI. The secondary objective was to evaluate efficacy based on urinary GAG levels and assessments of gross and fine motor function, cardiac function, vision, hearing, and health resource utilization. In addition, the safety of ERT in infants was evaluated at these two dose levels. We describe the efficacy and safety results of galsulfase therapy administered in four infants for a minimum of 52 weeks.

\section{Methods}

\section{Study design}

This was a multicenter, multinational, open-label, randomized, two-dose level study conducted in accordance with International Conference on Harmonization Good Clinical Practice (ICH GCP) and the principles of the Declaration of Helsinki. An institutional review board (IRB) or independent ethics committee (IEC) at each study center approved the study protocol, the subject informed consent form (ICF) and all subject recruitment materials. Parents or legal guardian signed an ICF prior to study enrollment and performance of any study-specific procedures and assessments.

Subjects who met eligibility criteria or received protocol exemptions at screening underwent baseline assessments over a 2-week period and were randomized 1:1 to weekly, openlabel treatment with either 1.0 or $2.0 \mathrm{mg} / \mathrm{kg}$ of galsulfase administered by IV infusion for a minimum of 52 weeks.

\section{Subject selection}

Subjects were initially required to be less than 1 year of age, to be diagnosed with MPS VI (through documented prenatal diagnosis or fibroblast or leukocyte ASB enzyme activity level $<10 \%$ of the lower limit of the normal range) and to have no evidence of skeletal dysplasia based on physical examination. Protocol exemptions were granted by the sponsor to the investigative sites for two infants based on age greater than 1 year (53 and 55 weeks) and for all subjects based on presence of skeletal dysplasia. Due to the rarity of MPS VI and difficulty in diagnosis before 2 to 3 years of age, recruitment was challenging. After significant effort ( $>2$ years) to recruit patients, it was deemed that inclusion of patients slightly over 1 year of age and with radiographic evidence of skeletal dysplasia was sufficient and necessary to complete the study. Two subjects (subjects 1 and 2) were identified and evaluated for MPS VI based on known family history (older sibling with diagnosed MPS). The other two subjects (subjects 3 and 4) were tested for MPS VI based on clinical symptoms.

Study drug administration

Subjects received weekly IV infusions of galsulfase. Two subjects (subjects 1 and 4) received pre-medication with antihistamine from the time of initiation of study drug infusions. Galsulfase doses were recalculated at monthly intervals based 
on the most recent weight obtained. Galsulfase solution was diluted with sterile $0.9 \%$ sodium chloride solution to $50 \mathrm{~mL}$ for subjects weighing less than $5 \mathrm{~kg}, 100 \mathrm{~mL}$ for subjects between 5 and $10 \mathrm{~kg}$ and $250 \mathrm{~mL}$ for subjects weighing at least $10 \mathrm{~kg}$. Infusion rates were adjusted so that approximately $2.5 \%$ of the total solution was infused during the first hour and the remaining volume (approximately $97.5 \%$ ) over a minimum of an additional $3 \mathrm{~h}$.

\section{Primary efficacy assessments}

Primary efficacy was assessed by changes in dysmorphic features in physical examinations, radiographic changes, and anthropometric growth measurements. Physical examinations were performed by individual site investigators at screening and follow-up visits, noting presence or absence of dysmorphic changes characteristic of MPS VI including, but not limited to, coarse facies, joint contractures, bony abnormalities of the spine and thorax, and claw hand deformity. Skeletal surveys were performed at baseline and at 52 weeks after initiation of study treatment. Radiographs were reviewed by a pediatric radiologist (R.S. Lachman) with extensive experience evaluating radiographs for MPS pathologies. Growth assessments, consisting of length, weight and head circumference measurements (Kuczmarski et al 2002), were conducted at baseline and throughout the duration of treatment for each patient. Because subjects were enrolled over a period of $>$ 2 years, growth data ranged from 52 weeks for the last subject enrolled to 152 weeks for the first subject enrolled.

\section{Secondary efficacy assessments}

Urinary GAG levels were evaluated at baseline and throughout the duration of treatment for each subject prior to infusions using the Automated Quantitation of Total Sulfated Glycosaminoglycans in Urine procedure with the GAGBotI Analyzer (Whitley et al 1989). Gross and fine motor skills were evaluated using the DENVER II Development Test (Frankenburg et al 1992) in three subjects (subjects 1, 2 and 4) and by the Griffiths Scales (Ivens and Martin 2002) in one subject (subject 3) at baseline and weeks 26 and 52.

Cardiac data were extracted from reports submitted by participating sites. At each site, cardiac function was assessed at baseline and at week 52 by transthoracic two-dimensional echocardiography including color and Doppler interrogation of cardiac valves. Measurements included left ventricular chamber dimensions in systole and diastole, diastolic septal and posterior wall thicknesses, and interrogation of cardiac valves for the presence or absence of stenosis or regurgitation. Body surface area-based Z-scores were calculated for chamber dimensions and wall thicknesses to allow comparison within the same subject over time and between subjects of different body surface areas (http://parameterz.blogspot.com/).
Complete ophthalmologic evaluations were conducted at baseline and at week 52, and included intraocular pressure (IOP) for glaucoma, examination of the retina and optic nerve, and documentation of corneal clouding. Audiological testing by audiometry was conducted at baseline and at week 52 . Health resource utilization at baseline and throughout the study was queried through a questionnaire which documented the occurrence of physical therapy, hospitalizations, procedures performed, outpatient visits, and emergency room (ER) visits.

\section{Safety assessments}

Safety was assessed throughout each infusion and for a minimum of $1 \mathrm{~h}$ post-infusion, by physical examinations, medical history, vital signs, concomitant medications (prescription and over the counter), changes in laboratory parameters, anti-rhASB antibody testing and adverse events (AEs). Total anti-rhASB antibody analysis was conducted using a validated bridged immunoassay format based on electrochemiluminescence (ECL) detection (BioMarin Pharmaceutical Inc., Novato, CA, USA) and was performed throughout the duration of treatment for each subject.

\section{Statistical methods}

Descriptive statistics were used to summarize the data in this small number of subjects. Categorical variables were summarized using frequencies, percentages, and shift from baseline. Continuous variables were summarized by the number of subjects, mean, median, standard deviations, minimum and maximum values.

\section{Results}

\section{Demographics}

Four subjects enrolled and received a minimum of 52 weeks of 1.0 or $2.0 \mathrm{mg} / \mathrm{kg} /$ week (two subjects per dose level) galsulfase treatment. No subject withdrew from the study. All subjects were male with a mean age at the time of enrollment of 9.2 months (40 weeks). At baseline, subjects 1, 2, 3, and 4 were aged 8.8, 3.3, 12.1 and 12.7 months, respectively. The two oldest subjects were randomized to $2.0 \mathrm{mg} / \mathrm{kg} / \mathrm{week}$ galsulfase. The diagnosis of MPS VI disease was confirmed upon enrollment (ASB enzyme activity in fibroblasts for 1 subject and leukocyte ASB enzyme activity in whole blood for 3 subjects). Serum TSH and free T4 levels were assessed as normal for all subjects. Urinary GAG levels at baseline were high for all subjects, with an overall mean of $870 \mu \mathrm{g} / \mathrm{mg}$ creatinine. Subjects 3 and 4 in the $2.0 \mathrm{mg} / \mathrm{kg} /$ week dose group were administered all doses of scheduled galsulfase infusions. Subjects 1 and 2 in the $1.0 \mathrm{mg} / \mathrm{kg} /$ week dose group each 
missed at least one galsulfase infusion (a total of four missed infusions) within the first 52 weeks of treatment, resulting in a compliance rate of $98 \%$ over this time period. In total, subjects received 392 of 396 scheduled doses over the course of the study for an overall treatment compliance rate of $99 \%$.

Primary efficacy

\section{Physical appearance}

All subjects presented with some characteristic clinical features of MPS VI at baseline and throughout the study period, as shown in Table 1. Kyphosis (subjects 1, 3 and 4), and chest wall deformities (pectus carinatum in subjects 1 and 3, and pectus excavatum in subject 4) were the most commonly reported findings at baseline. Facial dysmorphism improved in subject 3 and did not worsen in subject 2 after 52 weeks of ERT. Pectus carinatum in subject 3 was reportedly less pronounced after 52 weeks of therapy. Clinical kyphosis was absent at baseline but developed in subject 2 by week 26 . Macrocephaly $>97$ th percentile was noted in subject 3 at baseline and through week 52. Mild joint contractures, reported at baseline for subjects 1 and 2, did not appear to change in severity during the study period. Hepatosplenomegaly, which was present at baseline in subjects 1 and 4, had normalized after 52 weeks in subject 1 and did not worsen in subject 4 . An umbilical hernia persisted throughout the study in subject 3 and developed in subjects 2 and 4 by week 26 .

\section{Radiographic features}

Table 2 summarizes the radiographic findings at baseline and at week 52 for the four subjects. Some radiographic abnormalities were present in all subjects at baseline, including the youngest subject (subject 2) who was 3.3 months of age. The radiographic changes observed at 52 weeks reveal that galsulfase treatment did not prevent the progression of dysostosis multiplex in any of the subjects. The spine, pelvic and hand films of subject 1 (Fig. 1) are illustrative of the presentation and progression of some manifestations of dysostosis multiplex. By 52 weeks, all subjects demonstrated characteristic superiorly notched vertebral bodies, acetabular hypoplasia, and/or metacarpal shortening and proximal pointing. There was, however, radiographic improvement of kyphosis in subject 3. Radiographically, no untoward or unexpected changes in skeletal features attributable to galsulfase treatment were observed.

\section{Growth}

All subjects were assessed as normal for length at baseline and maintained normal length for the duration of the study, which ranged from 52 to 152 weeks depending on time of enrollment
(Fig. 2a). Normal weight gain was maintained throughout the study for subjects 2, 3 and 4 (Fig. 2b). Catch-up weight gain was observed for subject 1 , who had a screening weight below the 3rd percentile for his age of 9 months that steadily increased to the 10th-25th percentiles by week 52 and to the 25th-50th percentiles by study completion (Fig. 2b). Subjects 2, 3 and 4 had normal head circumference measurements at screening and growth remained within normal range for 52 weeks and beyond. Subject 1 had head circumference $>97$ th percentile from baseline through week 52 ; normal growth was attained by week 104 (head circumference between 95 th-97th percentile).

\section{Secondary efficacy}

\section{Urinary $G A G$}

At baseline, all four subjects had high urinary GAG levels consistent with MPS VI disease, with an overall mean of $870 \mu \mathrm{g} / \mathrm{mg}$ creatinine. All subjects experienced a urinary GAG level reduction of $\approx 70 \%$ by week 6 ; by week 52 , a mean decrease in GAG level of $75 \%$ was achieved (mean $=220 \mu \mathrm{g} / \mathrm{mg}$ creatinine) (Supplementary Figure 1). Formation of anti-rhASB antibodies did not appear to affect reductions in urinary GAG level for any of the subjects (Supplementary Figure 1). Urinary GAG concentrations remained low for the three subjects who were in the study for longer than 52 weeks.

\section{Gross and fine motor function}

Normal motor development was observed in subjects 2, 3 and 4 at baseline and throughout the study period. Motor delay was suspected in subject 1 at baseline and subsequent visits.

\section{Cardiac function}

Diastolic left ventricular chamber dimensions and posterior wall and septal thicknesses were within one standard deviation of normal (Z-scores ranging from 0.0025 to 0.8925 ) both at baseline and at week 52 of treatment (Supplementary Table 1). Mean shortening fraction for the group was normal both at baseline and at week 52. The incidence of mitral regurgitation decreased from 3 to 2 infants; aortic regurgitation was not identified. Neither aortic nor mitral stenosis was observed in these subjects.

\section{Vision and hearing}

All subjects had normal funduscopic examinations. Corneal clouding was observed at baseline for subjects 3 and 4 and persisted through study completion. Intraocular pressure for these two subjects was measured and found to be normal. 
Table 1 Physical characteristics of subjects $1-4$ at baseline, 26 weeks and 52 weeks as reported by individual site investigators

\begin{tabular}{|c|c|c|c|c|}
\hline & & Baseline & 26 weeks & 52 weeks \\
\hline \multirow[t]{11}{*}{ Subject 1} & General appearance & - Normal & - Normal & - Normal \\
\hline & Head, eyes, ears, nose, throat & - Normal & • Coryza & • Normal \\
\hline & Respiratory & • Normal & - Normal & - Normal \\
\hline & Cardiovascular & - Normal & • Normal & - Normal \\
\hline & \multirow[t]{2}{*}{ Gastrointestinal } & - Enlarged liver & - Liver size decreased & - Normal liver \\
\hline & & - Enlarged spleen & - Spleen size decreased & - Normal spleen \\
\hline & \multirow[t]{4}{*}{ Musculoskeletal } & • Kyphosis & - Kyphosis & • Kyphosis \\
\hline & & - Prominent last/lowest rib & - Prominent last/lowest rib & - Prominent last/lowest rib \\
\hline & & $\begin{array}{l}\text { - Mild contractures elbows, } \\
\text { knees }\end{array}$ & $\begin{array}{l}\text { - Mild contractures elbows, } \\
\text { knees }\end{array}$ & $\begin{array}{l}\text { - Mild contractures elbows, } \\
\text { knees }\end{array}$ \\
\hline & & - Mild pectus carinatum & - Mild pectus carinatum & - Mild pectus carinatum \\
\hline & Genitourinary & - Right inguinal hydrocele & - Hydrocele size decreased & $\begin{array}{l}\text { - Bilateral inguinal hernia } \\
\text { and right hydrocele }\end{array}$ \\
\hline \multirow[t]{10}{*}{ Subject 2} & General appearance & - Mild coarse facies, flat nasal bridge & - Mild coarse facies & - Mild coarse facies \\
\hline & Head, eyes, ears, nose, throat & - Mild coarse facies, flat nasal bridge & - Mild coarse facies & - Mild coarse facies \\
\hline & Respiratory & • Normal & • Normal & • Normal \\
\hline & Cardiovascular & - Normal & - Normal & - Normal \\
\hline & \multirow[t]{2}{*}{ Gastrointestinal } & - Diastasis recti & - Diastasis recti & - Diastasis recti \\
\hline & & & - Small umbilical hernia & - Small umbilical hernia \\
\hline & \multirow[t]{3}{*}{ Musculoskeletal } & - Mild knee flexion contracture & - Mild knee flexion contracture & - Mild knee flexion contracture \\
\hline & & • Bilateral talipes equinovarus & - Bilateral talipes equinovarus & - Bilateral talipes equinovarus \\
\hline & & & • Mild kyphosis & • Mild kyphosis \\
\hline & Genitourinary & - Normal & • Normal & • Normal \\
\hline \multirow[t]{8}{*}{ Subject 3} & General appearance & - Mild coarse facies & - Softening of facial features & - Softening of facial features \\
\hline & \multirow[t]{2}{*}{ Head, eyes, ears, nose, throat } & • Macrocephaly & - Less ENT infections & - Corneal clouding \\
\hline & & - Gingival hyperplasia & - Softer hair & \\
\hline & Respiratory & - Normal & - Normal & - Normal \\
\hline & Cardiovascular & - Systolic murmur 1/6 & - Systolic murmur 1/6 & - Systolic murmur 2/6 \\
\hline & Gastrointestinal & - Mild umbilical hernia & - Mild umbilical hernia & • Mild umbilical hernia \\
\hline & Musculoskeletal & - Mild kyphosis & - Mild kyphosis & - Pectus carinatum less pronounced \\
\hline & Genitourinary & • Normal & • Normal & - Normal \\
\hline \multirow[t]{10}{*}{ Subject 4} & General appearance & - MPS VI phenotype & - MPS VI phenotype & - MPS VI phenotype \\
\hline & Head, eyes, ears, nose, throat & • Normal & • Normal & • Normal \\
\hline & Respiratory & • Normal & • Normal & • Normal \\
\hline & Cardiovascular & • Normal & • Normal & • Normal \\
\hline & \multirow[t]{2}{*}{ Gastrointestinal } & - Palpable liver and spleen & - Palpable liver and spleen & - Palpable liver and spleen \\
\hline & & & - Umbilical hernia & - Umbilical hernia \\
\hline & \multirow[t]{3}{*}{ Musculoskeletal } & - Left valgus foot & - Left valgus foot & - Left valgus foot \\
\hline & & • Lumbar kyphosis & - Lumbar kyphosis & - Lumbar kyphosis \\
\hline & & - Pectus excavatum & - Pectus excavatum & - Pectus excavatum \\
\hline & Genitourinary & - Inguinal hernia & • Normal & • Normal \\
\hline
\end{tabular}

Table excludes reference to surgically placed PICC lines, MediPort, shunt lines, etc

Corneal clouding was not present at baseline but developed and progressed in subjects 1 and 2 .

All subjects had some hearing loss in both ears at baseline; bilateral deafness was reported in subject 4. At study completion, improvement in hearing in one ear was noted in subject 2 , while subjects 3 and 4 had demonstrated improvements in both ears.

\section{Health resource utilization}

All subjects required the use of health resources $(\geq 1$ treatment with antibiotics, concomitant medication, hospitalization, procedure performed, outpatient visit, or ER visit) during the study. Ear, nose, throat (ENT) and respiratory complications 
Table 2 Radiological characteristics of subjects 1-4 at baseline and at 52 weeks

\begin{tabular}{|c|c|c|c|}
\hline & & Baseline & 52 weeks \\
\hline \multirow[t]{9}{*}{ Subject 1} & Spine & - Superior notched L1 & - Superior notched L1 \\
\hline & & • Gibbus & - Increased gibbus \\
\hline & & & $\begin{array}{l}\text { - Marked subluxation of L1 over L2 } \\
\text { and slight T12 over L1 }\end{array}$ \\
\hline & Pelvis & - Rounded iliac wings & - Rounded iliac wings \\
\hline & & • Tapered lower ilia & - Increased tapered lower ilia \\
\hline & & - Normal acetabular roofs & - Poor acetabular roof formation \\
\hline & Hands and wrists & - Mild metacarpal shortening & - Continuing mild metacarpal shortening \\
\hline & & - No significant proximal pointing & • Mild proximal pointing \\
\hline & & & - Carpal ossification delay \\
\hline \multirow[t]{6}{*}{ Subject 2} & Spine & - Mild superior notched L1, L2 & - Mild superior notched T12 \\
\hline & & & - Moderate superior notched L1, L2 \\
\hline & Pelvis & - Poor acetabular roof formation & - Rounded iliac wings \\
\hline & & & - Tapered lower ilia \\
\hline & & & - Poor acetabular roof formation \\
\hline & Hands and wrists & - Normal & • Normal \\
\hline \multirow[t]{9}{*}{ Subject 3} & Spine & - Mild superior notched T12 & - Same superior notching of T12, L1, L2 \\
\hline & & - Severe superior notched L1, L2 & • Milder kyphosis \\
\hline & & • Kyphosis & \\
\hline & Pelvis & - Rounded iliac wings & - Rounded iliac wings \\
\hline & & - Tapered lower ilia & - Increased tapered iliac wings into acetabular roof \\
\hline & & - Normal acetabular roofs & - Poor acetabular roof formation \\
\hline & Hands and wrists & • Not available & - Mild metacarpal shortening \\
\hline & & & - Moderate proximal pointing \\
\hline & & & - Carpal ossification delay/small carpal centers \\
\hline \multirow[t]{10}{*}{ Subject 4} & Spine & - Severe superior notched L1 & • Unreadable film (overlying cast) \\
\hline & & - Moderate superior notched L2 & \\
\hline & & - Hypoplastic L1 vertebral body & \\
\hline & & • Gibbus & \\
\hline & Pelvis & - Rounded iliac wings & - Upper iliac wings obscured by cast \\
\hline & & - No tapering & - No tapering \\
\hline & & - Poor acetabular roof formation & - Poor acetabular roof formation \\
\hline & Hands and wrists & - Moderate metacarpal shortening & - Moderate metacarpal shortening \\
\hline & & - Mild proximal pointing & - Moderate proximal pointing \\
\hline & & - No carpal ossification delay/normal carpal sizes & - Mild carpal ossification delay/normal carpal sizes \\
\hline
\end{tabular}

(e.g., upper respiratory tract infections, otitis media, respiratory distress, nasal congestion) as well as fever were the most common problems to require medical intervention and were typically resolved through use of antibiotics and other medications. Subject 1 was hospitalized for pneumonia during week 3 of ERT and for respiratory distress at week 25 . Subject 3 was hospitalized for adenoid hypertrophy at week 9. Subject 4 underwent inguinal hernia repair at week 1.

\section{Safety}

No clinically significant physical findings related to safety were observed. Only one subject (subject 4) in the $2.0 \mathrm{mg} /$ $\mathrm{kg}$ /week dose group had clinically significant changes in vital signs. This subject experienced several events of pyrexia during and after infusions that were clinically significant and were reported as AEs.

No clinically significant abnormal laboratory values were reported during the study. The majority of test results were within the normal reference ranges and no systemic changes compromised subject safety. Subject 1 had the minimum quantifiable dilution factor (DF) anti-rhASB antibody titer (30 DF) at baseline; by week 6 , there were measurable ( $\geq 30$ DF) anti-rhASB antibody titers in all subjects. Subjects 2 and 3 developed total anti-rhASB antibody titers of $\geq 1,770,000 \mathrm{DF}$ (maximum quantifiable value) within 52 weeks of treatment 
Fig. 1 Radiographs of subject 1 at baseline and at 52 weeks. a Lateral spine, baseline: superior notched (inferiorly beaked) L1 vertebral body; significant gibbus. Lateral spine, 52 weeks: superior notched, hypoplastic L1; increased gibbus; multiple vertebral subluxations. b Pelvis, baseline: rounded iliac wings; tapered lower ilia (characteristic of dysostosis multiplex). Pelvis, 52 weeks: increased tapering of lower ilia into acetabular roof region; rounded iliac wings. c Hand and wrist, baseline: mild metacarpal shortening; no significant proximal pointing. Hand and wrist, 52 weeks: continuing mild metacarpal shortening, mild proximal pointing; carpal ossification delay with tiny carpal centers

\section{a}
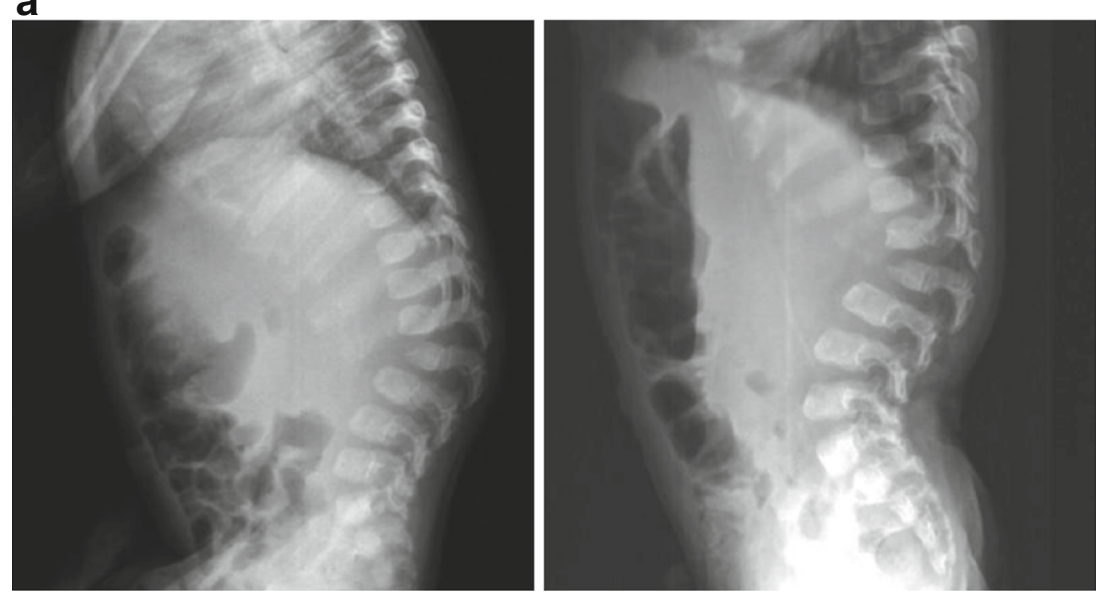

b
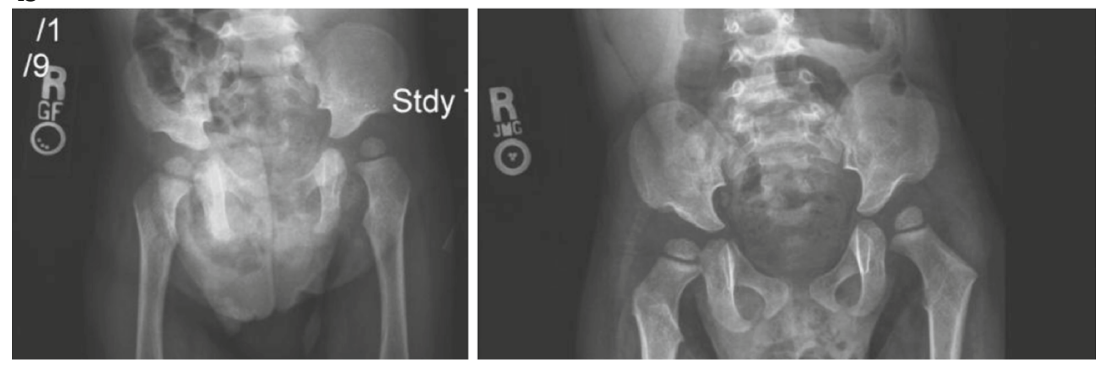

C

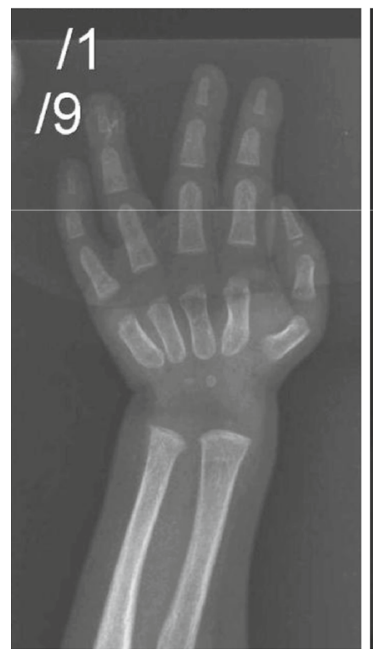

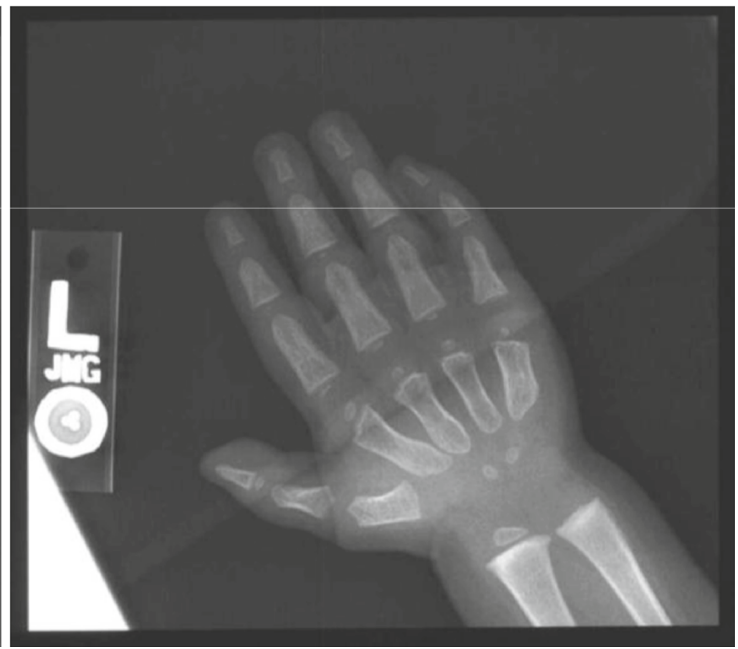

(Supplementary Figure 1). Subject 1, who was in the study for longer than 52 weeks, developed total anti-rhASB antibody titers of $\geq 1,770,000 \mathrm{DF}$ at week 78 ; by study termination these dropped to 590,490 DF. However, urinary GAG levels remained low compared with baseline levels for all subjects.

All four subjects experienced AEs during 52 weeks of treatment with galsulfase (Table 3). A total of 135 AEs were reported, of which 74 were reported for the subjects in the $1.0 \mathrm{mg} / \mathrm{kg} /$ week dose group (35 AEs for subject 1 and 39 AEs for subject 2) and 61 reported for those in the $2.0 \mathrm{mg} / \mathrm{kg} /$ week dose group
(16 AEs for subject 3 and 45 AEs for subject 4). Two subjects experienced possible treatment-related AEs (one event of nasal congestion in subject 2 and three events of fever for subject 4). Two subjects experienced a severe $\mathrm{AE}$ that was not related to treatment (cervical spinal stenosis in subject 1; bilateral deafness reported at baseline in subject 4). No subject discontinued from the study due to an AE or died during the study. Three subjects (subjects 2, 3 and 4) experienced a total of nine AEs during an infusion. Subject 4 had three events of fever that were considered to be possibly infusion-associated reactions (an $\mathrm{AE}$ that 
Fig. 2 a Length by age relative to age-matched percentages of normal values. ${ }^{1} \mathbf{b}$ Weight by age relative to age-matched percentages of normal values. ${ }^{1}$

${ }^{1}$ Reference curves are 3, 5, 10, $25,50,75,90,95$ and 97 percent of normal by age a

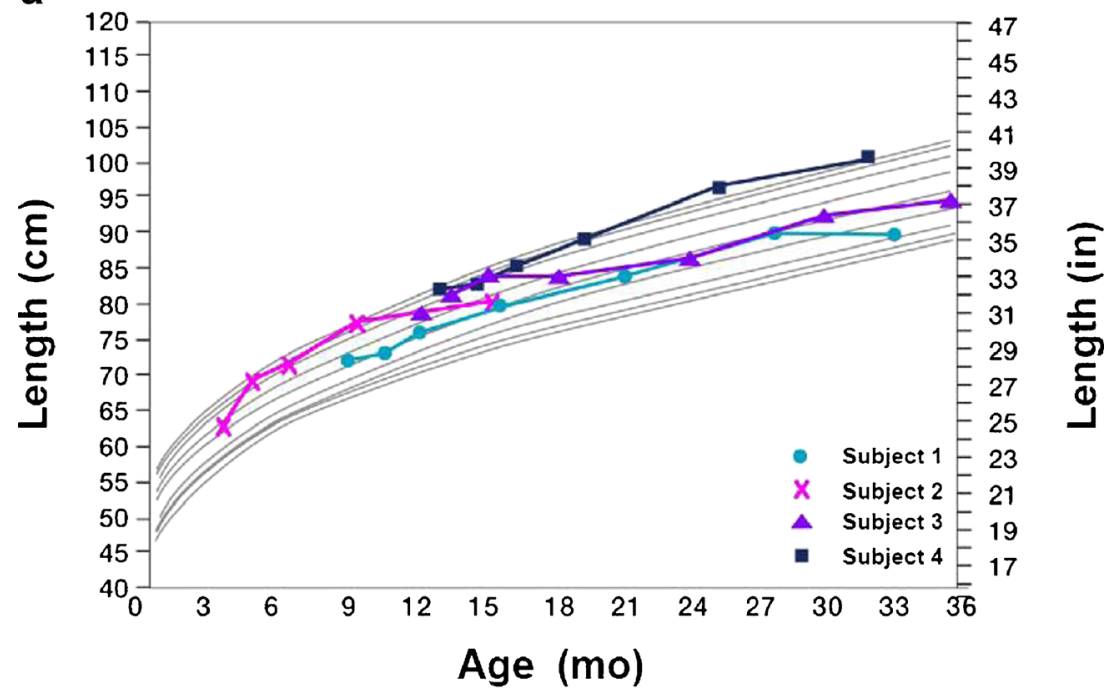

b

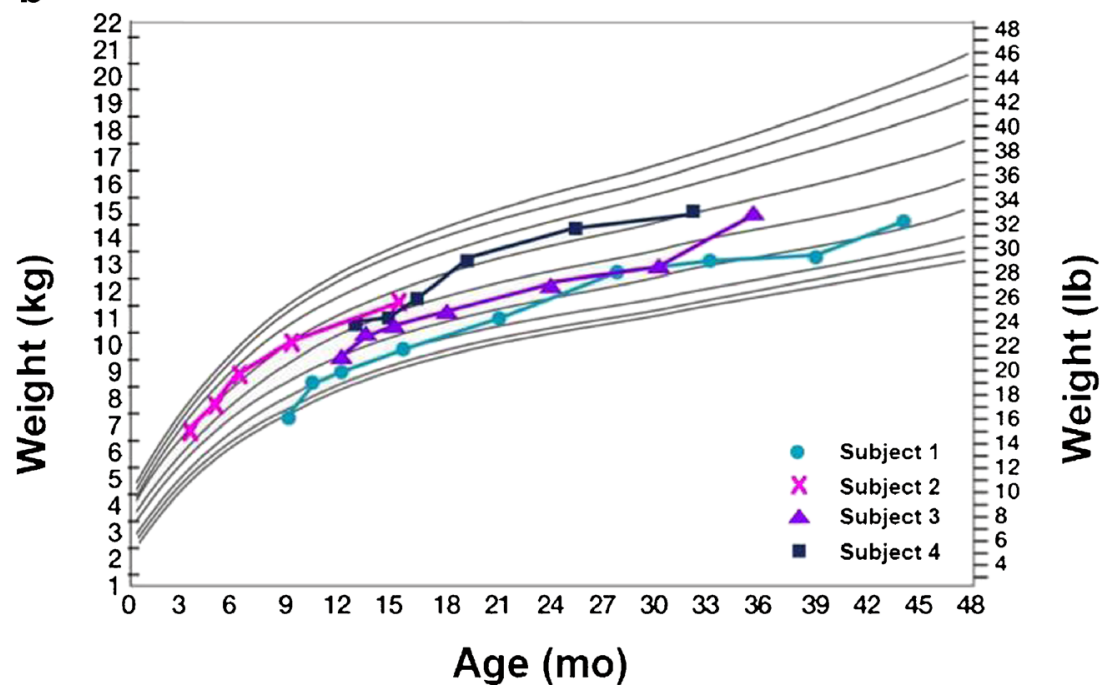

occurred during an infusion and was related to treatment with galsulfase); none were considered serious or severe, and the rate of subsequent infusions was not adjusted as a result of these infusion-associated reactions. Two of these fever events were resolved after treatment with paracetamol.

Although SAEs were more frequent in the high dose group (five versus three SAEs), none of the SAEs were identified by investigators as related to galsulfase infusion (Table 3). Of these, one was assessed as severe (bilateral deafness), six were moderate in severity (pneumonia, respiratory distress, adenoid hypertrophy, poor venous access, scoliosis, thoracic cast), and one was assessed as mild (inguinal hernia repair).

\section{Discussion}

The use of galsulfase therapy in MPS VI infants has not been extensively studied. The phase 1, 2, and 3 clinical trials did not include subjects younger than 5 years of age. Two recent sibling study reports suggest that galsulfase administered at the $1.0 \mathrm{mg} / \mathrm{kg} /$ week dose level in infants is safe and provides clinical benefit (Furujo et al 2011; McGill et al 2010). Although the limited number of subjects, short period of treatment, and absence of a control group or matched sibling population in our study do not allow for definitive conclusions regarding efficacy of galsulfase in this age group, results from this study do provide further insight into the potential benefits and limitations of early initiation of therapy.

The primary goal of this study was to investigate the effect of two dose levels of galsulfase on the progression of MPS VI skeletal dysplasia as determined by dysmorphic changes, radiographic changes, and growth. Our results suggest that galsulfase therapy improves or prevents progression of facial dysmorphism in infants, a finding that is consistent with the two MPS VI sibling studies (Furujo et al 2011; McGill et al 
Table 3 Summary of treatment-emergent adverse events during 52 weeks of galsulfase therapy

\begin{tabular}{|c|c|c|c|c|c|c|}
\hline & \multicolumn{2}{|c|}{$1.0 \mathrm{mg} / \mathrm{kg}$ dose $(n=2)$} & \multicolumn{2}{|c|}{$2.0 \mathrm{mg} / \mathrm{kg}$ dose $(n=2)$} & \multicolumn{2}{|c|}{$\begin{array}{l}\text { All subjects } \\
(n=4)\end{array}$} \\
\hline & Subject & Event $^{\mathrm{a}}$ & Subject & Event $^{\mathrm{a}}$ & Subject $^{\mathrm{a}}$ & Event $^{\mathrm{a}}$ \\
\hline & $n$ & $n$ & $n$ & $n$ & $n$ & $n$ \\
\hline Any AEs & 2 & 74 & 2 & 61 & 4 & 135 \\
\hline Study drug-related $\mathrm{AE}^{\mathrm{b}}$ & 1 & 1 & 1 & 3 & 2 & 4 \\
\hline Any SAEs & 2 & 3 & 2 & 5 & 4 & 8 \\
\hline Study drug-related $\mathrm{SAE}^{\mathrm{b}}$ & 0 & 0 & 0 & 0 & 0 & 0 \\
\hline Withdrawal from study due to $\mathrm{AE}$ & 0 & 0 & 0 & 0 & 0 & 0 \\
\hline Death & 0 & 0 & 0 & 0 & 0 & 0 \\
\hline AEs during infusion & 1 & 3 & 2 & 6 & 3 & 9 \\
\hline Study drug-related $\mathrm{AEs}^{\mathrm{b}}$ during infusion & 0 & 0 & 1 & 3 & 1 & 3 \\
\hline
\end{tabular}

${ }^{a}$ Multiple events were counted if a subject had the same AE with different onset dates or times

${ }^{\mathrm{b}}$ AEs classified by the investigator as possibly or probably related to study drug

2010). However, galsulfase treatment did not prevent the progression of dysostosis multiplex in the four subjects in this study, although radiographic improvement of kyphosis was observed in one subject. Similarly, dysostosis multiplex changes continued to develop in the galsulfase-treated infants in the MPS VI sibling studies. Despite initiation of ERT at 8 weeks and 182 weeks of treatment, the skeletal survey of the younger Australian MPS VI sibling was reported to reveal similar radiographic abnormalities at 3.6 years as those of her older sibling at the same age, who was untreated at the time (McGill et al 2010). Prevention of scoliosis in the younger sibling was associated with galsulfase treatment, however. Pre- and postERT spine and hand radiographs of the younger sibling of the Japanese MPS VI sibling pair revealed progression of manifestations of dysostosis multiplex despite commencement of therapy at 6 weeks and 36 months of treatment; however, these radiographic changes were comparatively milder than those of her older sibling at the same age, suggesting that galsulfase may have slowed the progression of the characteristic radiographic changes (Furujo et al 2011). In our study, randomization allocated the lower dose level to the two youngest subjects, subjects 1 and 2, who at baseline showed fewer radiographic abnormalities compared to the older subjects. The impact of a higher dose or an increased dosing frequency on bone pathology warrants further investigation. The impact of neonatal intervention should also be investigated. A newborn without radiological evidence of dysostosis multiplex was reported to have initiated galsulfase treatment at 5 days of age (Horovitz et al 2013); radiological follow-up of this patient would be insightful, particularly as our youngest subject (subject 2) already had radiographic abnormalities at onset of therapy at age 3.3 months. Elucidation of the mechanisms that cause the various bone pathologies of MPS disease may provide additional insight into the optimal mode of therapy.
All four subjects were able to maintain or achieve normal growth for the duration of the study. However, it is difficult to determine if treatment with galsulfase contributed to normal growth (length) maintenance since MPS VI patients may demonstrate growth acceleration with advanced bone maturation during the first years of life (Heron et al 2004); a similar growth pattern has also been reported in young children with Morquio A syndrome (Montano et al 2008). Longer follow-up over 5-10 years will be necessary to assess the long-term effects of galsulfase on height. Increased height and rate of growth in response to long-term galsulfase therapy has been demonstrated in MPS VI patients aged 5 years and older, with those under 16 years of age showing the greatest improvements (Decker et al 2010).

Consistent with results from previous clinical studies (Harmatz et al 2006, 2008), reductions of approximately $70 \%$ in urinary GAG levels from baseline were observed for all subjects (Supplementary Figure 1). These levels approached age-normalized upper limits of normal (Gallegos-Arreola et al 2000; Iwata et al 2000; Wood et al 2012). While urinary GAG levels decreased throughout the study, anti-rhASB antibodies increased to levels as high as $\geq 1,770,000 \mathrm{DF}$ (Supplementary Figure 1). A recent study of MPS VI patients followed for 5 years showed no correlation between antibody titers and urinary GAG levels, including in patients who developed titers $\geq 1,770,000 \mathrm{DF}$ (Hendriksz et al 2013). Similar stability of urinary GAG levels in the presence of antibody was also observed for most patients in the phase 3 clinical trial (Harmatz et al 2006).

No declines in gross or fine motor function were observed in any of the infants, including subject 1 with suspected motor delay. Motor development and speech delay have been reported in MPS VI children under 5 years of age (Horovitz et al 2013). It will require longer follow-up of the study cohort to determine 
the significance of the delay in subject 1 and the continued normality in the other subjects. It is notable that subject 1 had to relocate with one parent to the study site and this disruption may have contributed to the developmental delay.

Cardiac dimensions were within one standard deviation of normal and cardiac function was normal at both baseline and after 52 weeks of treatment in these infants. Although mitral regurgitation was identified in three subjects at baseline, it was observed in only two infants after 52 weeks. None of the subjects had aortic regurgitation, aortic stenosis or mitral stenosis. Our findings suggest that early initiation of treatment with galsulfase may prevent the development of ventricular hypertrophy and the progression of even mild valve regurgitation, findings that are hallmarks of MPS VI in older children (Braunlin et al 2013). Preservation of cardiac function was associated with early initiation of ERT in the MPS VI sibling studies (Furujo et al 2011; McGill et al 2010). Long-term ERT has been shown to prevent the progression of cardiac valve abnormalities when administered before the age of 12 years (Braunlin et al 2013).

Improvements in hearing were noted in three of our subjects, including bilateral improvement in subject 4 who had baseline bilateral deafness and was dosed at $2.0 \mathrm{mg} / \mathrm{kg} / \mathrm{week}$. Early initiation of galsulfase treatment was associated with improvement and prevention of hearing loss in the Japanese sibling study (Furujo et al 2011). However, early initiation of ERT was not found to be effective in preventing the progression of corneal clouding in our subjects, a finding that is consistent with the MPS VI sibling studies (Furujo et al 2011; McGill et al 2010). The avascular nature of the cornea likely reduces exposure of the cornea to circulating galsulfase.

Our results suggest that early initiation of galsulfase treatment may improve or prevent progression of hepatosplenomegaly, which is in line with findings from the two sibling studies (Furujo et al 2011; McGill et al 2010). Reductions in liver and spleen size following ERT have been observed in older MPS VI patients (Harmatz et al 2005; Hendriksz et al 2013). However, based on our study, early ERT does not appear to prevent the development of hernias.

All four subjects required the use of health resources during the study. Generally, health resources were used to address MPS VI disease manifestations and to treat illnesses common to children of the same age range of the subjects. The number of subjects is small and the absence of a control group makes it difficult to draw any conclusions about changes in health utilization with galsulfase.

Galsulfase was safe and well tolerated in the four subjects who participated in this study. Clinical changes were primarily indicative of MPS VI disease and were also similar between the two dose groups. In addition, there were no clinically relevant abnormal changes in laboratory test results over time for subjects in either group. One subject (subject 4) had clinically significant changes in vital signs which consisted of events of pyrexia that occurred during infusion and were considered possibly related to drug; however these reactions were not consistent with anaphylactoid-type reactions, resolved after three infusions and did not require any change in rate or any pre-infusion medication. The safety profile of the $2.0 \mathrm{mg} / \mathrm{kg} /$ week dose was not different in a clinically meaningful manner to that of the $1.0 \mathrm{mg} / \mathrm{kg} /$ week dose. The overall safety profile is consistent with that of previous galsulfase clinical trials involving subjects older than 5 years of age (Harmatz et al 2006, 2008).

No significant difference was observed in the clinical or safety outcomes of the two dose groups, although it is important to recognize that the groups are small. Since the two older subjects were randomized to the high dose group, it is possible that we did not observe a difference in efficacy because these subjects started therapy at a significantly later age. Furthermore, the subjects in the high dose group were diagnosed with MPS VI based on clinical symptoms, suggesting that they could have more rapidly progressing disease compared to the two subjects in the low dose group who were diagnosed based on known family history. It is unlikely that the lack of difference in efficacy between the two doses is related to high antibody levels since one subject in the high dose group (subject 4) had very low antibody titers while the other subject in the high dose group (subject 3 ) had similar antibody titers to those observed for the subjects receiving the lower dose (Supplementary Figure 1).

Further investigation of galsulfase therapy initiated early in subjects with MPS VI, including long-term follow-up of growth outcomes, is warranted to provide more complete information on the potential benefits of early treatment. The four subjects in this study are being followed to further elucidate long-term effects of early initiation of ERT. A Clinical Surveillance Program sponsored by BioMarin Pharmaceutical Inc. is currently underway to document the long-term effects of galsulfase treatment on clinical outcomes and safety in patients with MPS VI (Hendriksz et al 2013).

Acknowledgments The authors acknowledge the participation of study patients and their families and the expert assistance of all study site coordinators (Jo Ann Johnson and Jacqueline Madden, Oakland, CA USA; Nathalie Reynes, Lyon, France; Hesham Mahmoud, Los Angeles, CA, US) and study site personnel.

The clinical study was sponsored by BioMarin Pharmaceutical Inc (BioMarin). As the study sponsor, BioMarin was responsible for the design of the study and analysis of data and provided assistance with writing this report. This article reports the results of a clinical trial (registered at www.clinicaltrials.gov; NCT00299000). Authors not employed by the sponsor confirm independence from the study sponsor and their contributions were not influenced by the sponsor.

This study was supported, in part, with funds provided by the NIH CTSA grant UL1 RR0241315 (P. R. Harmatz). The content is solely the responsibility of the authors and does not necessarily represent the official views of the National Institutes of Health. 
Conflicts of interest P. R. Harmatz, N. Guffon, L. M. Randolph, E. Braunlin, and R. S. Lachman have provided consulting services to BioMarin Pharmaceutical Inc (BioMarin). P. R. Harmatz has received research grants, participated in advisory boards, and received speakers honoraria and travel support from BioMarin. P. Garcia has received travel support from BioMarin. L. M. Randolph has received research funding from BioMarin. E. Braunlin has participated in advisory boards and received speakers honoraria and travel support from BioMarin. R. Shediac and C. Decker are employees and stockholders of BioMarin Pharmaceutical Inc.

Open Access This article is distributed under the terms of the Creative Commons Attribution License which permits any use, distribution, and reproduction in any medium, provided the original author(s) and the source are credited.

\section{References}

Braunlin E, Rosenfeld H, Kampmann C et al (2013) Enzyme replacement therapy for mucopolysaccharidosis VI: long-term cardiac effects of galsulfase (Naglazyme(R)) therapy. J Inherit Metab Dis 36:385-394

Byers S, Nuttall JD, Crawley AC, Hopwood JJ, Smith K, Fazzalari NL (1997) Effect of enzyme replacement therapy on bone formation in a feline model of mucopolysaccharidosis type VI. Bone 21:425-431

Crawley AC, Niedzielski KH, Isaac EL, Davey RC, Byers S, Hopwood JJ (1997) Enzyme replacement therapy from birth in a feline model of mucopolysaccharidosis type VI. J Clin Invest 99:651-662

Decker C, Yu ZF, Giugliani R et al (2010) Enzyme replacement therapy for mucopolysaccharidosis VI: growth and pubertal development in patients treated with recombinant human $\mathrm{N}$-acetylgalactosamine 4sulfatase. J Pediatr Rehabil Med 3:89-100

Frankenburg WK, Dodds J, Archer P, Shapiro H, Bresnick B (1992) The Denver II: a major revision and restandardization of the Denver Developmental Screening Test. Pediatrics 89:91-97

Furujo M, Kubo T, Kosuga M, Okuyama T (2011) Enzyme replacement therapy attenuates disease progression in two Japanese siblings with mucopolysaccharidosis type VI. Mol Genet Metab 104:597-602

Gallegos-Arreola MP, Machorro-Lazo MV, Flores-Martinez SE et al (2000) Urinary glycosaminoglycan excretion in healthy subjects and in patients with mucopolysaccharidoses. Arch Med Res 31:505-510

Giugliani R, Harmatz P, Wraith JE (2007) Management guidelines for mucopolysaccharidosis VI. Pediatrics 120:405-418

Harmatz P, Ketteridge D, Giugliani R et al (2005) Direct comparison of measures of endurance, mobility, and joint function during enzymereplacement therapy of mucopolysaccharidosis VI (MaroteauxLamy syndrome): results after 48 weeks in a phase 2 open-label clinical study of recombinant human $\mathrm{N}$-acetylgalactosamine 4sulfatase. Pediatrics 115:e681-e689

Harmatz P, Giugliani R, Schwartz I et al (2006) Enzyme replacement therapy for mucopolysaccharidosis VI: a phase 3, randomized, double-blind, placebo-controlled, multinational study of recombinant human $\mathrm{N}$-acetylgalactosamine 4-sulfatase (recombinant human arylsulfatase B or rhASB) and follow-on, open-label extension study. J Pediatr 148:533-539

Harmatz P, Giugliani R, Schwartz IV et al (2008) Long-term follow-up of endurance and safety outcomes during enzyme replacement therapy for mucopolysaccharidosis VI: final results of three clinical studies of recombinant human $\mathrm{N}$-acetylgalactosamine 4-sulfatase. Mol Genet Metab 94:469-475

Hendriksz CJ, Giugliani R, Harmatz P et al (2013) Design, baseline characteristics, and early findings of the MPS VI (mucopolysaccharidosis VI) Clinical Surveillance Program (CSP). J Inherit Metab Dis 36:373-384

Heron D, Baumann C, Benichou JJ, Harpey JP, Le Merrer M (2004) Early diagnosis of Maroteaux-Lamy syndrome in two patients with accelerated growth and advanced bone maturation. Eur J Pediatr 163: 323-326

Horovitz DD, Magalhaes TS, Acosta A et al (2013) Enzyme replacement therapy with galsulfase in 34 children younger than five years of age with MPS VI. Mol Genet Metab 109:62-69

Ivens J, Martin N (2002) A common metric for the Griffiths Scales. Arch Dis Child 87:109-110

Iwata S, Sukegawa K, Kokuryu M et al (2000) Glycosaminoglycans in neonatal urine. Arch Dis Child Fetal Neonatal Ed 82:F78

Kuczmarski RJ, Ogden CL, Guo SS et al (2002) 2000 CDC Growth Charts for the United States: methods and development. Vital Health Stat 11:1-190

Lachman R, Martin KW, Castro S, Basto MA, Adams A, Teles EL (2010) Radiologic and neuroradiologic findings in the mucopolysaccharidoses. J Pediatr Rehabil Med 3:109-118

McGill JJ, Inwood AC, Coman DJ et al (2010) Enzyme replacement therapy for mucopolysaccharidosis VI from 8 weeks of age-a sibling control study. Clin Genet 77:492-498

Montano AM, Tomatsu S, Brusius A, Smith M, Orii T (2008) Growth charts for patients affected with Morquio A disease. Am J Med Genet A 146A:1286-1295

Swiedler SJ, Beck M, Bajbouj M et al (2005) Threshold effect of urinary glycosaminoglycans and the walk test as indicators of disease progression in a survey of subjects with Mucopolysaccharidosis VI (Maroteaux-Lamy syndrome). Am J Med Genet A 134A: $144-150$

Thumler A, Miebach E, Lampe C et al (2012) Clinical characteristics of adults with slowly progressing mucopolysaccharidosis VI: a case series. J Inherit Metab Dis 35:1071-1079

Valayannopoulos V, Nicely H, Harmatz P, Turbeville S (2010) Mucopolysaccharidosis VI. Orphanet J Rare Dis 5:5

Whitley CB, Ridnour MD, Draper KA, Dutton CM, Neglia JP (1989) Diagnostic test for mucopolysaccharidosis. I. Direct method for quantifying excessive urinary glycosaminoglycan excretion. Clin Chem 35:374-379

Wood T, Bodamer OA, Burin MG et al (2012) Expert recommendations for the laboratory diagnosis of MPS VI. Mol Genet Metab 106:73-82 